\title{
Teratoma: masa anexial en mujeres jóvenes
}

Teratoma: adnexal mass in young women

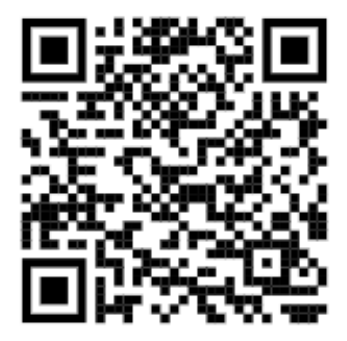

${ }^{1}$ Médico general, graduada de la Universidad de Ciencias Médicas (UCIMED) Código médico: 15306. catacoto1493@hotmail.com

${ }^{2}$ Médico general, graduado de la Universidad de Ciencias Médicas (UCIMED) Código médico: 15663. miimenezvi18@gmail.com

${ }^{3}$ Médico general, graduada de la Universidad Latina de Costa Rica (U.Latina) Código médico: 15569. dra.snaranjo@gmail.com
${ }^{1}$ Dra. Catalina Coto Chaves
Instituto de psicopedagogía integral colegio Issac Martin (IPICIM), San José, Costa Rica
Solumed - Hospital La California, San José, Costa Rica
$\frac{{ }^{2} \text { Dr. Mauricio Jiménez Víquez }}{\text { https://orcid.org/0000-0003-4392-5591 }}$
${ }^{3}$ Dra. Stephanie Naranjo Alfaro

Investigadora independiente, San José, Costa Rica

(i) https://orcid.org/0000-0001-9293-0616
RECIBIDO

05/04/2019
CORREGIDO
20/04/2019
ACEPTADO

$01 / 05 / 2019$

\section{RESUMEN}

Las masas ováricas, de trompas uterinas y de tejidos conectivos aledaños, se conocen como masas anexiales y son un problema ginecológico común. Pueden encontrarse en todas las edades y existen diferentes subtipos. Los más comunes en mujeres premenopáusicas son los tumores de células germinales, de los cuales el teratoma maduro es el más frecuente. Al evaluarlas, siempre deben descartarse emergencias quirúrgicas y signos de malignidad. El ultrasonido es el método radiológico de elección para estudiar una masa anexial. Esta revisión bibliográfica se enfoca en el abordaje inicial de una masa anexial y la diferenciación por ultrasonido de lesiones benignas y malignas. Cuando se sospechan lesiones malignas o en pacientes muy sintomáticas, el abordaje es quirúrgico y con el objetivo de preservar la fertilidad.

PALABRAS CLAVE: teratoma; ovario; anexos uterinos; quiste dermoide; neoplasias ováricas.

\section{ABSTRACT}

Ovarian, uterine tube masses, and adjacent connective tissue tumors, are known as adnexal masses and are a common gynecologic problem. They can be found at any age and there is a wide variety of subtypes. Germ cell tumors are the most common among premenopausal women, specially the mature teratoma. The evaluation goal is to discard surgical emergencies and malignancy. Ultrasound is the radiologic gold standard method to study an adnexal mass. This article is focused on 
the initial approach to the patient with an adnexal mass and the differentiation between benign and malignant lesions based on the ultrasound findings. If a malignant tumor is suspected or the patient is symptomatic, fertility preserving surgery is recommended.

KEYWORDS: teratoma; ovary; adnexa uteri; dermoid cyst; ovarian neoplasms.

\section{INTRODUCCIÓN}

Las masas ováricas, de tubas uterinas, mesovarium, mesosalpinx, de estructuras proximales del tracto urinario, intestino $y$ tejido conectivo adyacente, son conocidas como masas anexiales (1). Esta revisión bibliográfica se enfoca en el abordaje inicial de masas anexiales en mujeres premenopáusicas jóvenes, principalmente en los teratomas, que son el tumor más frecuente entre los 10 y 30 años de edad y pueden ser encontrados frecuentemente de manera incidental. Pese a que en esta población la mayoría son benignas, existen también lesiones malignas y lesiones que pasan desapercibidas y crecen durante algunos años hasta que provocan los primeros síntomas. Esto aumenta el riesgo de torsión, ruptura y diseminación. La importancia de la sospecha de estas lesiones radica en su diagnóstico temprano, en la caracterización a través del ultrasonido y en caso de malignidad en estadíos tempranos, que la paciente pueda beneficiarse de la cirugía y quimioterapia.

Es de vital importancia conocer las diferencias ultrasonográficas entre masas benignas y malignas para orientar el diagnóstico y poder referir adecuadamente al paciente a ginecología para un seguimiento más adecuado.

\section{EPIDEMIOLOGÍA}

La mayoría de masas anexiales tienen su origen en los ovarios. Se estima que las mujeres tienen un $5-10 \%$ de riesgo de ser sometidas a un procedimiento quirúrgico en su vida por sospecha de una neoplasia ovárica (1). La posibilidad de presentar un cáncer de ovario en la vida es de $1.8 \%$ y de este porcentaje, $70 \%$ tiene enfermedad avanzada al diagnóstico debido a la escasa sintomatología que se presenta en estadíos tempranos (2). El cáncer de ovario es la neoplasia ginecológica maligna más frecuente en menores de 25 años. El ovario incluye diversos tipos de tejido. Las neoplasias más comunes son las epiteliales, pero también existen tumores de células germinales y del estoma de los cordones sexuales. Los tumores de células germinales son el tipo más frecuente en mujeres entre los $10 \mathrm{y}$ los 30 años (3).Algunos factores de riesgo para el desarrollo de cáncer de ovario son la edad, raza blanca, infecundidad, uso de estimulantes de la fecundidad, antecedente de cáncer de mama, colon 0 endometrio y la exposición a radiación (4).

\section{PRESENTACIÓN}

- Premenopausia: la mayoría de masas anexiales ocurre en mujeres 
en edad reproductiva, incluyendo a adolescentes postmenárquicas, $\mathrm{y}$ tienden a ser benignas (1). El riesgo de desarrollar una neoplasia ovárica maligna en esta edad es del $6-11 \%$. Los tumores de células germinales, son los más frecuentes y constituyen el $70 \%$ delas neoplasias en este grupo (3). Los tipos más comunes de neoplasias en edad reproductiva, son cistadenoma, tumor de Brenner y teratoma quístico maduro (3).

- Postmenopausia: la malignidad, es más frecuente en la postmenopausia. De las mujeres postmenopáusicas mayores a 50 años, al menos 30\% de las masas ováricas son malignas y la mayoría son carcinomas de tipo epitelial (3).

\section{EVALUACIÓN INICIAL}

Durante el abordaje inicial, se deben realizar una adecuada historia clínica y examen físico. Las pacientes típicamente se presentan con síntomas ginecológicos, o bien, asintomáticas con una masa observada de manera incidental en un ultrasonido de rutina. EI síntoma más común es el dolor o sensación de presión pélvica o abdominal.

Otras se presentan también con distensión abdominal, síntomas gastrointestinales, urinarios o sangrado uterino anormal (1).

Al realizar la historia clínica es importante preguntar por las características del dolor, si éste comenzó súbitamente, su duración, si es constante o intermitente, si está o no relacionado con el ciclo menstrual, si es posible que esté embarazada, si hay presencia de fiebre o descarga vaginal, si existen antecedentes heredofamiliares de enfermedad pélvica y cáncer de ovario, mama, útero y colon debido al riesgo aumentado de desarrollar cáncer de ovario en caso de mutaciones en los genes BRCA ó Síndrome de Lynch (1).

En casos de masa anexial asociada a dolor severo y de inicio súbito, fiebre y descargas vaginales, sangrado uterino en primer trimestre $\mathrm{e}$ inestabilidad hemodinámica, es primordial descartar condiciones como embarazo ectópico, absceso tubo-ovárico, torsión anexial, quiste ovárico roto y otras condiciones consideradas emergencias médicas ó quirúrgicas (1).

Si durante el examen físico no se palpan masas, esto no descarta su existencia, pero si se percibe, deben notarse su tamaño, consistencia, movilidad, si hay ascitis o distensión abdominal. Algunos datos sugestivos de malignidad son la presencia de una masa sólida, fija, irregular y nodular. También se debe observar si hay signos de virilización o hirsutismo, ya que algunas masas anexiales son estimuladas por hormonas reproductivas (1). En casos de sospecha de malignidad, es fundamental explorar sitios de posibles focos primarios que podrían hacer metástasis a ovario. Algunos de estos órganos son el estómago, colon, recto, mama y endometrio que al diseminarse a ovario producen el conocido tumor de Kruckenberg. El estómago y las mamas son los órganos que más frecuentemente hacen metástasis a ovario.

Asimismo, se exploran también potenciales sitios de metástasis de cáncer de ovario, como lo son el recto, hígado, bazo, pulmón, nódulos inguinales o supraclaviculares (1). 


\section{DIAGNÓSTICO POR IMÁGENES}

La ecografía es un procedimiento no invasivo y de alta sensibilidad. Cabe recalcar su accesibilidad y bajo costo en comparación con la tomografía y la resonancia magnética, ya que en nuestro país, estos últimos poseen un costo elevado, su disponibilidad como estudios de rutina es limitado y son considerados de segunda línea en caso de no obtener resultados concluyentes con el ultrasonido (4).

El ultrasonido transvaginal es el estudio de imagen de elección para evaluar una masa anexial (1). En la mayoría de pacientes, se realiza uno transabdominal y uno transvaginal. El transabdominal es mejor tolerado y es más efectivo para valorar procesos abdominales. El transvaginal, brinda una mejor resolución de estructuras pélvicas y no requiere que la vejiga esté distendida. El principal objetivo del estudio por imágenes es caracterizar la masa para ver si es benigna 0 maligna. Sin embargo, el diagnóstico histológico definitivo se alcanza tras una exploración quirúrgica y toma de biopsia (1).

Pasos para la caracterización de una masa anexial por ultrasonido:

1. Determinar si es un quiste simple: Un quiste simple por lo general presenta fluido anecóico dentro de la cavidad, paredes delgadas y sombra acústica distal conservada, es decir, que la transmisión del sonido a través de la masa no se altera y no se pierde la señal de los tejidos detrás del quiste (2).

En mujeres premenopáusicas, quistes anexiales simples de $3-5 \mathrm{~cm}$ de diámetro, generalmente son fisiológicos y la Sociedad de Radiólogos en Ultrasonido no recomienda su seguimiento. Si están asintomáticas y presentan un quiste simple entre $5-7 \mathrm{~cm}$, se les realiza un ultrasonido control por año; pero si el tumor excede los $7 \mathrm{~cm}$, se sugiere realizar una resonancia magnética si no se logró detallar adecuadamente el aspecto del quiste (2).

Por otro lado, en mujeres postmenopáusicas, se usa un umbral de $3 \mathrm{~cm}$ para decidir dar seguimiento (2).

2. Si la masa no es un quiste simple, se deben descartar procesos como la involución del cuerpo lúteo, la presencia de 2 quistes simples unidos o un quiste hemorrágico que simulan tener septos 0 nódulos intramurales y buscar características de malignidad (2). La consistencia sólida de la masa es el dato de mayor peso pronóstico de malignidad (4). Ver TABLA 1.

3. El teratoma maduro, puede tener características de malignidad confundibles, como lo es la presencia de un nódulo hiperecóico dentro de la masa, este hallazgo, en especial si presenta sombra acústica, es un fuerte indicador de teratoma. También, los teratomas suelen ser uni-formemente hiperecóicos o tienen ecos lineales o punteados brillantes; ocasionalmente contie-nen nivel fluido-fluido, si el fluido ecogénico es no dependiente, es predictivo de teratoma (aun-que esto pasa en una minoría de casos). Además pueden presentar calcificaciones de diferentes tamaños, y aunque son poco comunes, la presencia de glóbulos flotantes son predictivos(5).

Cualquier masa con características de teratoma debe de ser evaluada con ultrasonido dippler a color, ya que la 
TABLA 1. Características ultrasonográficas de masas anexiales

\begin{tabular}{|c|c|}
\hline Benignas & Malignas \\
\hline $\begin{array}{ll}\text { - } & \text { Quiste unilocular de cualquier tamaño } \\
\text { - } & \text { No sólida ó sólida menor a } 7 \mathrm{~mm} \\
\text { - } & \text { Lesión de bordes regulares ó irregulares } \\
\text { - } & \text { Solgados y menor a } 10 \mathrm{~cm} \text { de diámetro } \\
\text { - } & \text { Ausencia de flujo sanguíneo }\end{array}$ & $\begin{array}{ll}\text { - } & \text { Tumor sólido irregular mayor a } 10 \mathrm{~cm} \\
\text { - } & \text { Ascitis } \\
\text { - } & \text { Tabiques, paredes gruesas, vegetaciones } \\
\text { - } & \text { Flujo elevado al doppler a color }\end{array}$ \\
\hline
\end{tabular}

presencia de flujo en la masa hace que el diagnóstico de teratoma maduro sea poco probable, e incrementa la posibilidad de teratoma inmaduro (maligno), struma ovarii u otra causa. En casos de lesión indeterminada o inconclusa porque la masa presenta características tanto benignas como malignas, se decide dar seguimiento con ultrasonido, otras técnicas de imagen y marcadores tumorales (5).

Históricamente se ha considerado que masas de mayor tamaño, tienen mayor riesgo de malig-nidad, no obstante, esto no se ha confirmado, de manera que un quiste simple de $10 \mathrm{~cm}$, por ejemplo, no es de mayor riesgo que un quiste de 2 $\mathrm{cm}$ con componente sólido y nodular (2).

\section{CLASIFICACIÓN}

\section{TUMORES DE CÉLULAS GERMINALES}

Representan el $20-25 \%$ de todas las neoplasias de ovario, pero son los tumores más comunes en mujeres entre los 10 y los 30 años de edad, constituyendo el $70 \%$ de todos las neoplasias en esta edad y solamente $5 \%$ son malignos. Se clasifican en teratomas, disgerminomas, tumores del saco vitelino, y coriocarcinomas no gestacionales (2). Estos tumores, crecen rápidamente en comparación con las neoplasias epiteliales y la mayoría se diagnostica en estadíos tempranos limitados al ovario (IA). Usualmente producen hormonas, particularmente unidad sub-beta de la hormona gonadotropina coriónica humana (en el caso del coriocarcinoma y algunos disgerminomas) y alfa feto proteína en el caso de tumores del saco vitelino y teratomas inmaduros. Tienden a ser masas grandes con una media de $16 \mathrm{~cm}$, de manera que $85 \%$ de las afectadas presenta dolor abdominal y sensación de masa. También pueden causar distensión abdominal, $20 \%$ se acompaña de ascitis, $20 \%$ presenta ruptura al diagnóstico y $5 \%$ asocia riesgo de torsión (2).

\section{TERATOMAS}

Teratoma deriva de la palabra griega "Teraton", que significa monstruo. Este tér-mino fue acuñado y usado por primera vez por Rudolph Virchow, padre de la patología moderna en 1863 en su libro sobre tumores (6).

Los teratomas comprenden el $95 \%$ de los tumores de células germinales y son los tumores no epiteliales benignos más frecuentes $(7,8)$.

Se dividen en 4 categorías: teratomas maduros, teratoma inmaduro, teratoma de transformación maligna y teratoma monodérmico altamente especializado 
(2).Dentro de las complicaciones, las más comunes son la infección y torsión. La ruptura y la transformación maligna son más infrecuentes (9). De igual manera, pueden asociarse a manifestaciones sistémicas o síndromes paraneoplásicos como anemia hemolítica autoinmune y encefa-litis por anticuerpos contra los receptores de N-metil-Daspartato (NMDAR) (10).

- Teratoma maduro: se conoce también como quiste dermoide. Es quístico y está compuesto por tejidos maduros diferenciados.

Constituye más del $95 \%$ de todos los teratomas $y$ tiende a ser benigno $y$ unilateral, sin embargo $10-15 \%$ pueden ser bilaterales. Es la neoplasia ovárica más frecuente en mujeres entre 20 y 30 años (2).

Poseen elementos de las 3 capas germinales: ectodermo, mesodermo y endodermo. De esta manera, pueden presentar tejidos diferenciados como piel, cabello, glándulas sebáceas, músculo, pulmón y de los sistemas urinario $y$ gastrointestinal (3). A nivel macroscópico, se ve como una masa multiquística con pelo, dientes y piel, mezclados con material sebáceo grueso, pegajoso y mal oliente.

En cuanto a la clínica, la mayoría de mujeres, se muestra asintomática y si presenta síntomas, están más relacionados al ta-maño alcanzado por la masa (2).

El teratoma maduro, es a su vez, la masa anexial más frecuente en el embarazo, generalmente durante la gestación son asintomáticas y son diagnosticadas de manera incidental tras un ultrasonido obstétrico de rutina (11).

\section{- Teratoma inmaduro: es}

conocido como teratoma maligno, teratoblastoma ó teratoma embrionario. Constituye apenas un $1 \%$ de todos los tumores de células germinales. Tiene diferentes grados de tejidos inmaduros y su clasificación histológica se basa en el grado de tejido neural inmaduro. Son los únicos tumores de células germinales que son histológicamente estadiados, desde grado I (bien diferenciado) hasta grado III (pobremente diferenciado). El grado histológico es un importante indicador de riesgo de diseminación extraovárica y de recurrencia. Pueden secretar alfa fetoproteína o lactato deshidrogenasa (2). La afectación del ovario contralateral es rara y por lo tanto no es necesaria una biopsia de rutina del ovario no involucrado, aunque en $10 \%$ de los casos, puede haber un teratoma benigno en el ovario contralateral. La metástasis a distancia de un teratoma inmaduro de ovario es poco frecuente (11).

- Teratomas con transformación maligna: la transformación maligna ocurre en $0.2-2 \%$ de los teratomas maduros. Aunque cualquiera de sus componentes puede malignizar, lo más común es que se desarrolle un carcinoma epidermoide 0 de células escamosas.

También pueden formarse carcinomas de células basales, melanomas, adenocarcinomas, sarcomas y carcinoma de tiroides. Los factores de riesgo para esta transformación son la edad mayor a 45 años, diámetro del tumor mayor a $10 \mathrm{~cm}$, que presente un rápido crecimiento y un ulltrasonido sospechoso de malignidad y en caso de demostrarse la transformación, el tratamiento debe 
dirigirse hacia la histología transformada (2).

- Teratomas monodérmicos altamente especializados: los más comunes son el struma-ovarii y el carcinoide, que es una neoplasia neuroendocrina. Generalmente son unilaterales. El struma-ovarii está compuesto por tejido tiroideo maduro, y la secreción de hormona tiroidea provoca hipertiroidismo clínico en $25-35 \%$ de los casos.

La mayoría son benignos (2). Los tumores carcinoides son raros, se asemejan a otros tipos gastrointestinales o respiratorios y secretan polipéptidos y aminas (entre ellos la serotonina), que producen principalmente flushing $y$ diarrea, aunque se han descrito casos de hipoglicemia hiperinsulinémica $(2,10)$.

\section{ESTADIAJE}

Los tumores malignos de células germinales son estadiados por la International Federation of Gynecology and Obstetrics (FIGO).

En general, estadío I involucra únicamente ovarios, estadío II se extiende a otros tejidos pélvicos, estadío III se extiende más allá de la pelvis o a nódulos linfáticos retroperitoneales pero sigue confinado a abdomen, y ya estadío IV, presenta metástasis distales 0 afectación hepática (2).

Tumores de alto grado y estadío tienden a ser más recurrentes, usualmente en forma de teratoma maduro (18).

\section{TRATAMIENTO Y MARCADORES TUMORALES}

Tradicionalmente, el manejo ha sido quirúrgico. La cirugía está indicada cuando la masa es sintomática, si hay sospecha de malignidad, si hay riesgo asociado de torsión o infección, si hay hallazgos sugestivos de metástasis en estudios radiológicos y cuando el tumor ha crecido más de $10 \mathrm{~mm}$ durante los primeros dos ultrasonidos de seguimiento a los 3 y 6 meses $(12,13)$.

La cirugía es generalmente una salpingoooferectomía unilateral. Si el tumor es grado I y limitado al ovario, no requiere más tratamiento, pero para estadíos más avanzados, se recomienda también quimioterapia coadyuvante con un protocolo de bleomicina, etopósido y cisplatino. La quimioterapia mejoró drásticamente el pronóstico. Así, la sobrevida a 5 años puede variar de $91 \%$ para estados I y II a 56\% para estadíos avanzados y recurrentes $(9,7)$.

En mujeres gestantes y no gestantes, el tratamiento es el mismo, siempre teniendo en consideración que al ser mujeres jóvenes, se debe tratar de preservar la fertilidad y que el riesgo de malformaciones asociadas a la quimioterapia en el primer trimestre es mayor (14).

El abordaje laparoscópico se considera el "gold standard" y la pieza debe ser extraída preferiblemente a través de la cicatriz umbilical a menos que la masa sea muy grande (15).

Cuando la sospecha de malignidad es baja pero no ha sido completamente excluida, se pueda acudir a una vigilancia con ultrasonido y marcadores tumorales (12). El uso de marcadores tumores es de mayor provecho en la postmenopausia. El Ca-125 es el marcador tumoral más usado para la detección de cáncer epitelial de ovario. Valores de Ca-125 por encima de $35 \mathrm{U} / \mathrm{ml}$ tienen una sensibilidad de $69-97 \%$ y una 
especificidad de $81-93 \%$. En premenopausia, se mide Ca-125 únicamente si hay hallazgos sospechosos de malignidad, porque en estas mujeres la sensibilidad $y$ especificidad es más baja, incluso de 50 $60 \%$ ya que existen otras condiciones ginecológicas, pulmonares, urinarias, otras malignidades y alteraciones del tracto gastrointesti-nal, que pueden elevarlo. Por esta razón se ha propuesto usar valores mayores a $200 \mathrm{U} / \mathrm{ml}$ en premenopáusicas, pero aún hay pocos estudios al respecto (12). El teratoma inmaduro puede secretar alfa feto proteína y lactado deshidrogenasa (11). El antígeno de carcinoma de células escamosas (SCC) es el marcador tumoral más útil para predecir la transformación maligna, sin embargo es positivo solamente en $30-67 \%$ de los casos, y su utilidad se sigue discutiendo (18).

\section{CONCLUSIÓN}

El cáncer de ovario es la malignidad ginecológica más frecuente en menores de 25 años. En mujeres jóvenes premenopáusicas, los tumores de células germinales, principalmente los teratomas maduros, son los más comunes. Aunque es cierto que el riesgo de malignidad es mayor en la postmenopausia, no puede descartarse que se presente en mujeres jóvenes y es de vital importancia detectar las masas anexiales de manera temprana y descartar su malignidad, ya que en muchos casos, no provocan síntomas y pasan desapercibidos mucho tiempo antes de ser diagnosticados en etapas tardías. La meta de la sospecha temprana, es valorar las características de la masa a través de un ultrasonido y distinguir entre características benignas y malignas para decidir si se le da seguimiento o si se debe tomar una biopsia para obtener el diagnóstico definitivo. Lastimosamente no existe un método poco invasivo para la toma de biopsias ováricas, de manera que en caso de sospecha de carcinoma, la paciente debe someterse a una intervención quirúrgica y si el tumor se encuentra en estadíos tempranos, puede responder adecuadamente a la cirugía y quimioterapia. Aunque la mayoría de teratomas son benignos, pueden crecer, provocar síntomas molestos y una minoría maligniza. El uso de marcadores tumorales muestra mayor beneficio en mujeres postmenopáusicas en las que los carcinomas epiteliales son más comunes. Su uso en la premenopausia aún se discute.

\section{REFERENCIAS}

1. Muto M. Approach to the patient with an adnexal mass [Internet]. Waltham (MA): UpToDate Inc; 2019 [Updated $2018 \quad$ Feb 23, cited 2019 March 29]. Available from:https://www.uptodate.com/contents/approach-to-the-patient-with-an-adnexal-mass

2. Gershenson D. Ovarian germ cell tumors: Pathology, clinical manifestations and diagnosis [In-ternet]. Waltham (MA): UpToDate Inc; 2019 [Updated 2017 Nov 30, cited 2019 March 29]. Available from:https://www.uptodate.com/contents/ovarian-germ-cell-tumors-pathology-clinical-manifestations-anddiagnosis 
3. Hoffman M, Hochberg L. Differential diagnosis of the adnexal mass [Internet]. Waltham (MA): UpToDate Inc; 2019 [Updated 2019 March 20, cited 2019 March 29]. Available from:https://www.uptodate.com/contents/differential-diagnosis-of-the-adnexal-mass

4. Gomez M. Ultrasonografía en la identificación de masas anexiales. Revista médica de Costa Rica y $\begin{array}{llll}\text { Centroamérica. } & \text { 2016; } & \text { 73(619): } & \text { Disponible }\end{array}$ en:http://www.binasss.sa.cr/revistas/rmcc/619/art36.pdf

5. Patel M. Ultrasound Differentiation of benign versus malignant adnexal masses [Internet]. Waltham (MA): UpToDate Inc; 2019 [Updated 2018 Nov 08, cited 2019 March 29]. Available from:https://www.uptodate.com/contents/ultrasound-differentiation-of-benign-versus-malignant-adnexal$\underline{\text { masses }}$

6. Gregory K, Gatie M. Mechanisms Regulating Stemness and Differentiation in Embryonal Carcinoma Cells. Stem Cells International. 2017; ID 3684178. https://doi.org/10.1155/2017/3684178

7. Dan V, Zacharias R, Martins V, Genaro A, et al. Teratoma ovariano imaturo gigante com te-ratoma maduro bilateral: relato do caso. Medicina (Ribeirao Preto). 2016; 49(5): 483489.https://doi.org/10.11606/issn.2176-7262.v49i5p483-489

8. Cabezas M, Rodríguez E, Rodríguez I, Márquez F, et al. Teratoma ovárico maduro e inma-duro a propósito de un caso. Ginecología y Obstetricia de México. 2017; 85(5), 331-337. Disponible en:http://www.scielo.org.mx/scielo.php?script=sci abstract\&pid=S030090412017000500331\&lng=es\&nrm=iso

9. Jorge S, Jones N, Chen L, Hou J, Tergas A, Burke W et al. Characteristics, treatment and out-comes of women with immature ovarian teratoma. Gynecologyc Oncology. 2016; 142(2): 261266.https://dx.doi.org/10.1016\%2Fj.ygyno.2016.05.024

10. Hamada A, Yamaka T, Tokunaga K, Moribata Y, Himoto Y, Kido A, et al. A comprehen-sive review of ovarian teratomas. Electronic Presentation Online System. 2016; C122.1.http://dx.doi.org/10.1594/ecr2016/C-1221

11. Madrigal A, Rosenkraz B. Teratoma inmaduro(maligno) de ovario. Medicina legal de Costa Rica. 2018; 35(1): 1409-1415. Disponible en: http://www.scielo.sa.cr/pdf/mlcr/v35n1/1409-0015-mlcr-35-01-146.pdf

12. Muto M. Management of an adnexal mass [Internet]. Waltham (MA): UpToDate Inc; 2019 [Updated 2018 Sep 24, cited 2019 March 29]. Available from: https://www.uptodate.com/contents/management-of-anadnexal-mass

13. Pascual A, Graupera B, Pedrero C, Rodríguez I, Ajossa S, guerrero S, et al. Long term results for expectant management of ultrasonographically diagnosed benign ovarian terato-mas. Obstet-rics and gynecology. 2017; 130(6): p.1244-1250. https://doi.org/10.1097/AOG.0000000000002327

14. Hasdemir P, Guvenal T, Mnekse S, Solmaz U, Kandiloglu A, Koyuncu F, et al. Ovarian Im-mature teratoma detected during pregnancy. Medical Science and discovery. 2016; 3(1): 16.http://dx.doi.org/10.17546/msd.39076

15. Sinha A, Ewies A. Ovarian mature cystic teratoma: Challenges of surgical management. In: Coleman R, editor. Obstetrics and Gynecology International. 2016; ID 2390178.http://dx.doi.org/10.1155/2016/2390178 